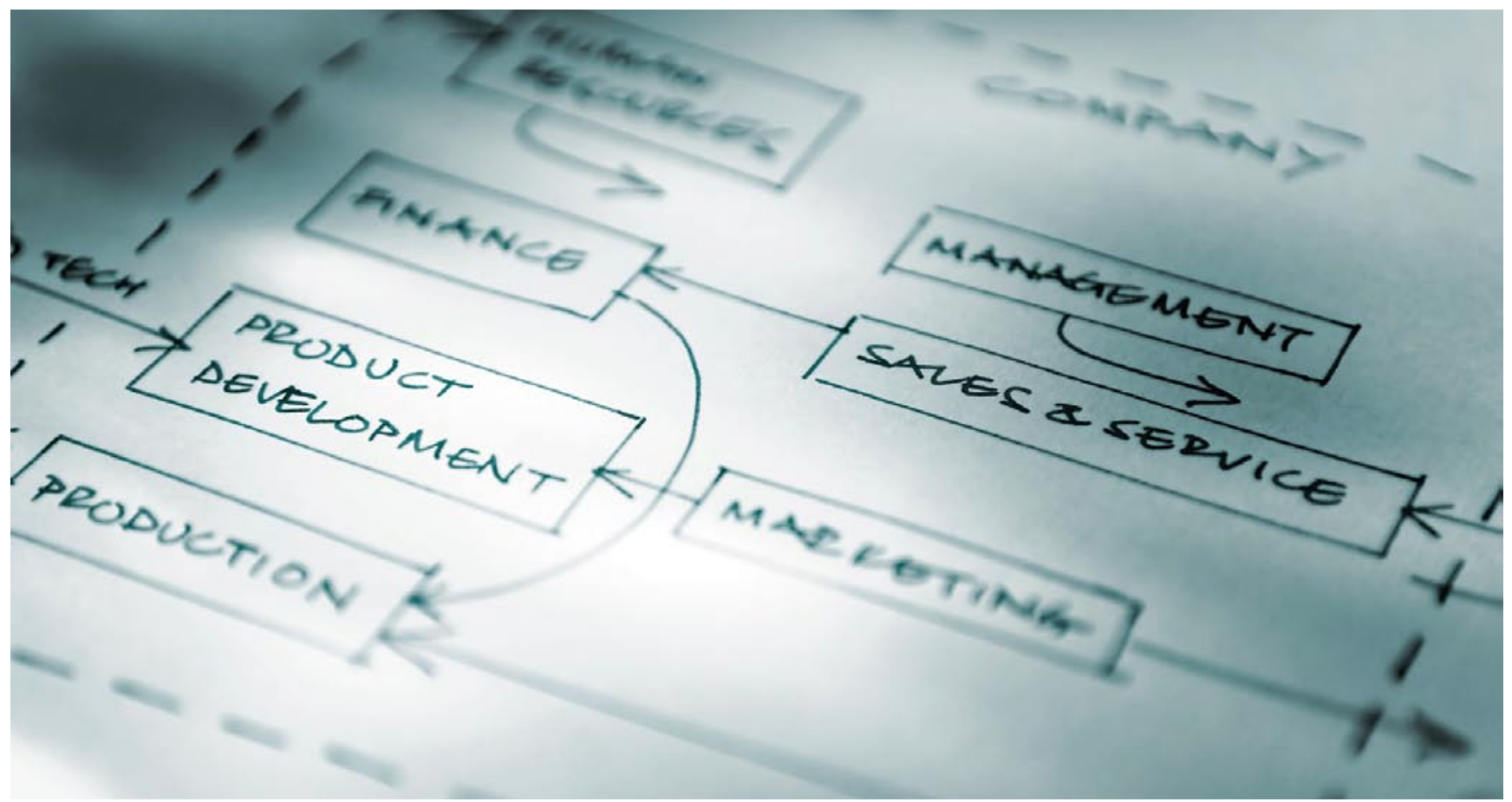

\title{
Organisationale Fähigkeiten für Dienstleistungsstrategien
}

Kürzere Technologiezyklen, steigende Wettbewerbsintensität auf globalen Märkten, erhöhter Druck auf Produktmargen und zunehmende Homogenisierung von Produkten führen viele Hersteller von Investitionsgütern zu einer strategischen Neuorientierung und dem Ausbau des Dienstleistungsgeschäfts. Für die erfolgreiche Positionierung als Entwicklungs- oder Outsourcingpartner benötigen Firmen jedoch bestimmte organisationale Fähigkeiten.

THOMAS FISCHER | HEIKO GEBAUER | ELGAR FLEISCH

$H$ ersteller von Investitionsgütern wandeln sich zunehmend von produktorientierten Unternehmen zu Unternehmen, die sich auf den Vertrieb von Leistungssystemen, d. h. Bündeln aus Produkt und Dienstleistungen konzentrieren (Belz et al. 1997, Gummesson 1994). GF AgieCharmilles, ein international führender Hersteller von Funkenerosionsmaschinen, hat sich erfolgreich von einem Produktverkäufer zu einem Anbieter von Leistungssystemen weiterentwickelt. GF AgieCharmilles ergänzt Produkte durch eine Vielzahl von Dienstleistungen. Hierzu gehören Logistikdienstleistungen im Bereich Ersatz- und Verschleißteile bzw. Verbrauchsmaterial, Dienstleistungen zur Unterstützung der Instandhaltungsaktivitäten der Kunden sowie der Privilege Club. Hauptziel der Ausweitung des Dienstleistungsgeschäfts sind verbesserte Kundenbindung, neue Möglichkeiten für den Verkauf von
Maschinen sowie zusätzliche Umsätze durch Querverkauf von Verbrauchsmaterialien.

Zwei Stoßrichtungen bei der strategischen Neuorientierung des Dienstleistungsgeschäfts bei Investitionsgüterherstellern sind die Veränderung zum Entwicklungspartner (EP) oder zum Outsourcingpartner (OP) (Gebauer et al. 2006). Ein erfolgreiches Beispiel für einen EP ist der Automobilzulieferer Magna-Steyr, der Automobilherstellern Entwicklungsdienstleistungen anbietet und z. B. für BMW den X3 entwickelt hat. Werkzeughersteller Hilti, der mit seinem Flottenmanagement für Bauwerkzeuge die gesamte Gerätebewirtschaftung seiner Kunden übernimmt, steht stellvertretend für die erfolgreiche Entwicklung zu einem OP. Beispiele wie das des Lackieranlagenherstellers Dürr, der letztlich daran scheiterte, sich zum OP für Automobilhersteller zu entwickeln und sein 
Dienstleistungsgeschäft verkaufte, zeigen jedoch, dass die Veränderung der Dienstleistungsstrategie nicht einfach ist. Ziel dieses Beitrags ist es aufzuzeigen, wie Unternehmen, die sich erfolgreich vom Investitionsgüterhersteller zum Entwicklungs- oder Outsourcingpartner verändert haben, ihr Organisationsdesign der Strategieänderung angepasst haben und welche organisationalen Fähigkeiten dazu notwendig sind.

\section{Investitionsgüterhersteller entwickeln sich zu Anbietern für Entwicklungs- und Outsourcingdienstleistungen}

Die strategischen Stoßrichtungen im Dienstleistungsbereich sind eng gekoppelt mit den Kundenaktivitäten und dem eigentlichen Dienstleistungsangebot für die Kundenaktivitäten. Entwicklungspartner (EP) bieten externen Kunden ihre Entwicklungskompetenz als Dienstleistung an und führen Entwicklungsaktivitäten für diese aus. Die Verantwortung für den gesamten Entwicklungsprozess verbleibt in der Regel beim Kunden. Mittels wissensintensiver Dienstleistungen wie kundenindividueller Konstruktion oder unterstützenden F \& E-Tätigkeiten positionieren sich EP als strategischer Partner des Kunden. Aus Sicht eines traditionellen Investitionsgüterunternehmens bedeutet dies ein Wandel vom Verkauf einer Produktionsanlage zur Entwicklung des gesamten Produktionsprozesses gemeinsam mit dem Kunden. Die Produktionsanlage ist dann nur noch ein Teil des entwickelten Produktionsprozesses. Bei der Erbringung einer Entwicklungsdienstleistung wird spezifisches Wissen zwischen Kunden und EP ausgetauscht. Dieser Wissensaustausch ist ein Alleinstellungsmerkmal beim Kunden und schafft einen signifikanten Wettbewerbsvorteil.

Outsourcingpartner (OP) übernehmen die Verantwortung für die Durchführung eines kompletten Kundenprozesses und verändern damit ihr Geschäftsmodell. Ein OP offeriert Kunden die Möglichkeit, ausschließlich für die erbrachte Leistung im übernommenen Kundenprozess zu bezahlen. Ein Beispiel hierfür ist ein Betreibermodell. Bei einem Betreibermodell übernehmen Firmen den Betrieb der eigenen Anlage und verrechnen nur noch die erbrachte Leistung (Lay/Schneider 2001). Zwei Ausprägungsformen dieses auch Performance Contracting genannten Konzepts sind der Verkauf einer Leistung und der Verkauf eines garantierten Leistungsergebnisses. Beim Leistungsverkauf vermietet der Hersteller das Kernprodukt inklusive einer produktbezogenen Dienst- leistung für einen bestimmten Zeitraum und Festpreis. Er garantiert folglich die Übernahme bestimmter Teile des Kundenprozesses. Beim Leistungsergebnisverkauf übernimmt der Hersteller das komplette Betreiberrisiko vom Kunden (Backhaus/Kleikamp 2001, Kleikamp 2002, Kleinaltenkamp et al. 2004) bei in der Regel leistungsabhängiger Bezahlung. Beispiele sind etwa ein Pumpenhersteller, der seinen Kunden anstelle der Pumpe die Leistung von $\mathrm{x} \mathrm{m}^{3} / \mathrm{s}$ transportierte Flüssigkeit verkauft, oder Dürrs Vorstoß, lackierte Autos anstelle von Lackieranlagen zu verkaufen, die Übernahme der Verantwortung für die Instandhaltung von Investitionsgütern und der Verkauf von garantierter Verfügbarkeit. Durch die Übernahme der Instandhaltung werden die fixen Instandhaltungskosten des Kunden reduziert.

\section{Wechsel der Dienstleistungsstrategie verlangt organisationale Veränderungen und spezifische organisationale Fähigkeiten}

Die erfolgreiche Veränderung der Dienstleistungsstrategie erfordert die Umsetzung einer spezifischen Konfiguration des Organisationsdesigns und hängt davon ab, wie mit den zugrundeliegenden organisationalen Fähigkeiten Wettbewerbsvorteile erzielt werden können (Neu/Brown 2005). Für die Entwicklung von vertriebsorientierten Dienstleistungsstrategien sind vor allem organisatorische Veränderungen hinsichtlich Unternehmenskultur, Organisationsstruktur und Personalmanagement erforderlich. In Übereinstimmung von Literatur und Praxisbeispielen lassen sich diese Dimensionen in einzelne organisatorische Elemente ausdifferenzieren (z. B. Homburg et al. 2003, Noch 1995, Matthyssens/Vandenbempt 1998). Die Unternehmenskultur beinhaltet die Elemente Wertverständnis für Dienstleistungen und Rollenverständnis der Mitarbeiter. Die Organisationsstruktur lässt sich durch die organisatorische Trennung des Produktund Dienstleistungsgeschäfts und die Sichtbarkeit der Dienstleistungsaktivitäten für den Kunden beschreiben. Das Personalmanagement umfasst Personalrekrutierung, -entwicklung und -entlohnung. Für eine erfolgreiche Umsetzung der Strategie müssen Unternehmen je nach Stoßrichtung bestimmte organisationale Fähigkeiten entwickeln, um diese Faktoren genau spezifizieren und anschließend zielgerichtet implementieren zu können.

\section{Tab. 1 Strategische Stoßrichtungen für Investitionsgüterhersteller}

\begin{tabular}{|c|c|c|}
\hline & Entwicklungspartner & Outsourcingpartner \\
\hline Wertversprechen für Kunden & $\begin{array}{l}\text { - Gemeinsame Entwicklung kundenindividueller Prozesse } \\
\text { - Kunden profitieren von Entwicklungskompetenzen } \\
\text { - Verkürzte Entwicklungszeiten und -kosten bei } \\
\text { gleichzeitig hoher Qualität }\end{array}$ & $\begin{array}{l}\text { - Übernahme der Verantwortung für die Durchführung } \\
\text { eines kompletten Kundenprozesses } \\
\text { - Reduzierte Kapitalbindung } \\
\text { - Kontinuierliche Verbesserung der übernommenen } \\
\text { Prozesse }\end{array}$ \\
\hline Dienstleistungsangebot & $\begin{array}{l}\text { - Forschungs- und Entwicklungsdienstleistungen } \\
\text { - Konstruktionsdienstleistungen }\end{array}$ & $\begin{array}{l}\text { - Übernahme von Kundenprozessen } \\
\text { (z. B. Instandhaltung, Logistik, Betreibermodelle, ...) }\end{array}$ \\
\hline
\end{tabular}




\section{Unternehmenskultur}

Für die Mitarbeiter aus F\&E verlangt der Wandel zum EP, ein Wertverständnis als Dienstleister für externe Kunden zu entwickeln. An die Stelle der eigenen Produktentwicklung als maßgebliche Quelle für Innovationen rücken gemeinsam mit den Kunden produzierte oder entwickelte Lösungen. Dienstleistungen als langfristiges Kundenbindungselement und Voraussetzung für zusätzlichen Umsatz und Profitabilität prägen vermehrt das Wertverständnis eines EP. Für die Entwicklung des Wertverständnisses als EP sind aus organisationaler Sicht Kompetenzen zum Erkennen der marketingbezogenen, finanziellen und strategischen Möglichkeiten für das Anbieten von F\&E-Dienstleistungen erforderlich. Das sind insbesondere Fähigkeiten für das Erfassen, Filtern und Verarbeiten von Informationen wie Kunden- und Marktdaten und für die Kommunikation des neuen Wertverständnisses innerhalb des Unternehmens.

Bei der Erbringung von F\&E-Dienstleistungen nehmen die Mitarbeiter die Rolle eines technischen Beraters für den Kunden ein. Technische Berater müssen ein umfassendes Verständnis für die Kundenprozesse entwickeln, um gemeinsam mit den Mitarbeitern des Kunden innovative Lösungen für dessen Prozesse zu erarbeiten. Im Vordergrund steht ein kontinuierlicher Lernprozess zur Sicherung einer optimalen Entwicklungs- und Konstruktionsleistung für den Kunden durch die Interaktion mit dem Kunden (Pine et al. 1995). Die detaillierten Kenntnisse, die F\&E-Mitarbeiter dabei über die Kundenprozesse gewinnen und die Kenntnisse, die der Kunde über die direkten Entwicklungskompetenzen des Herstellers erwirbt, setzen hohes Vertrauen, eine starke Orientierung zur Zusammenarbeit und eine generelle Lernkultur voraus (Möl- ler 2006). Diese starke Ausrichtung auf den Kunden hat den positiven Nebeneffekt der Kundenbindung und trägt somit zur Schaffung einer strategischen Partnerschaft bei.

Das Wertverständnis eines typischen OP entspricht einem reinen Dienstleister, der seinen Kunden die vollständige Übernahme von Kundenprozessen offeriert und ihnen ein bestimmtes Leistungsergebnis und eine vordefinierte Leistungsqualität garantiert. Kunden werden das Betreiben ihrer Prozesse nur dann einem OP überlassen, wenn der OP deutliche Kostenvorteile garantiert. OP begeben sich somit in einen harten Preiswettbewerb und fokussieren sich auf das Erzielen von Effizienz und Skaleneffekten durch standardisierte Dienstleistungen, während der Kunde nur am Ergebnis interessiert ist. Mitarbeiter des OP sind somit meist gefordert, ihr Wertverständnis als Anbieter individuell auf den Kunden zugeschnittener Dienstleistungspakete zum Anbieter von standardisierten, effizient zu erbringenden Dienstleistungen zu ändern. Für den Wandel zum OP sind einerseits Kompetenzen zum Erkennen der marketingbezogenen, finanziellen und strategischen Möglichkeiten von Outsourcingdienstleistungen und andererseits insbesondere der Risiken einer Übernahme von Kundenprozessen erforderlich. Dazu gehören die Fähigkeiten, Kundenprozesse gemäß Kundenbedürfnis betreiben zu können, standardisierte Dienstleistungen effizient erbringen zu können, die volle Verantwortung vom Kunden zu übernehmen und dieses Risiko kalkulieren zu können. Fähigkeiten zur Informationsverarbeitung sind Grundvoraussetzung.

Mitarbeiter aus dem Kundenkontakt eines OP müssen mehrere Rollen erfüllen. Gegenüber dem Kunden müssen sie als zuverlässige Betreiber der Anlagen in den Kundenprozessen auf-

\section{Tab. 2 Fähigkeiten zur Anpassung der Unternehmenskultur}

Entwicklungspartner

Elemente

Wertverständnis für Dienstleistungen

\section{Rollenverständnis}

Ausprägungen

- Dienstleister für externe Kunden sein

- Wertschöpfung durch gemeinsam mit den Kunden entwickelte Lösungen - Dienstleistungen als langfristiges Kundenbindungselement
Fähigkeiten

- Erkennen des Potenzials von F\&E-Dienstleistungen - Informationsverarbeitung - Kommunikation des neuen Wertverständnisses innerhalb des Unternehmens

-

\begin{tabular}{l} 
Ausprägungen \\
\hline - Reiner Dienstleister für \\
Kunden sein \\
- Dienstleistungen als Mittel \\
zur Lieferung des garantier- \\
ten Leistungsergebnisses \\
- Fokus auf das Erzielen von \\
Effizienz und Skaleneffekten
\end{tabular}

Outsourcingpartner

\begin{tabular}{l} 
Fähigkeiten \\
\hline - Erkennen des Potenzials \\
von Outsourcingdienst- \\
leistungen \\
- Erkennen der Risiken einer \\
Übernahme von Kunden- \\
prozessen \\
- Kundenprozesse gemäß \\
Kundenbedürfnis betreiben \\
- Standardisierte Dienst- \\
leistungen höchst effizient \\
erbringen \\
- Verantwortung übernehmen \\
- Risiko kalkulieren \\
- Informationsverarbeitung \\
- Verstehen der Kunden- \\
bedürfnisse in Bezug auf \\
übernommene Prozesse \\
- Anwendungskompetenzen \\
- Komplexitätsmanagement
\end{tabular}

- Zuverlässiger Anlagen-
betreiber
- Gleichzeitiges Erbringen
standardisierter Dienstleis-
tungen und eines kom-
plexen Dienstleistungs-
prozesses

\begin{tabular}{ll}
\hline - Technischer Berater & - Verstehen der Kunden- \\
- Entwickeln eines kontinuier- & prozesse \\
lichen Lernprozesses durch & - Kundenorientierung \\
Interaktion mit Kunden & - Vertrauen \\
- Leiten der gemeinsamen & - Orientierung zur \\
Entwicklung von Kunden- & Zusammenarbeit \\
prozessen & - Generelle Lernkultur
\end{tabular}


treten und gleichzeitig in der Lage sein, standardisierte Dienstleistungen effizient zu erbringen und den Kunden die garantierte Leistung aus einem komplexen Dienstleistungserbringungsprozess zu liefern. Die Mitarbeiter benötigen ein tiefes Verständnis der Kundenbedürfnisse in Bezug auf die übernommenen Prozesse und entsprechende Anwendungskompetenzen, um die garantierte und vom Kunden erwartete Leistung zu erbringen und etwaigen Problemen und Unregelmäßigkeiten zu begegnen. Weiterhin sind Kompetenzen im Umgang mit Komplexität unerlässlich, um die Vielfalt von Aufgaben in Bezug auf Produktfeatures, Betreiben der Prozesse und Erbringen von Dienstleistungen zu meistern. Insgesamt wird der Wandel in der Unternehmenskultur bei einem OP meist durch die Übernahme von Mitarbeitern des Kunden bei Übernahme von Kundenprozessen unterstützt.

\section{Organisationsstruktur}

EP definieren meist ein spezielles Team innerhalb der zentralen F\&E für die Vermarktung und Erbringung von Entwicklungsdienstleistungen. Die organisatorischen Grenzen zwischen dem speziellen Team und bestehender F\&E sind relativ durchlässig. So findet ein regelmäßiger Wissensaustausch statt, der z. B. durch Job-Rotation-Ansätze zwischen beiden Bereichen unterstützt wird. Die Fähigkeit zu einem systematischen Wissensmanagement ist ein wichtiger Erfolgsfaktor für EP. Der bestehende Entwicklungsbereich profitiert von dem Wissensaustausch auf Grund des Zugangs zu aktuellen Kundenprojekten bzw. zu technologischen Entwicklungen auf der Kundenseite. Unterstützt wird dies durch regelmäßig angesetzte Treffen mit Kunden und gemeinsam genutzte IT-Systeme. Das spezielle Entwicklungs- team wiederum hat durch den Wissensaustausch ausreichend Kenntnis über die neuesten technischen Entwicklungen innerhalb des eigenen Unternehmens. Für seine externen Kunden muss ein EP gut sichtbare Kontaktpunkte für sein Angebot an Entwicklungsdienstleistungen schaffen und klare interne Verantwortlichkeiten für das Dienstleistungsgeschäft regeln. Innerhalb des speziellen Entwicklungsteams sorgen klar geregelte Verantwortlichkeiten für einzelne Kunden für den Aufbau einer langfristigen und persönlichen Kundenbeziehung. Feste Meetingstrukturen und gemischte Teamzusammensetzungen während Entwicklungsprojekten fördern zusätzlich die Zusammenarbeit mit dem Kunden und helfen, ein umfassendes Bild der Kundenherausforderungen bei der Prozessentwicklung zu erhalten. Dazu ist es erforderlich, dass die Mitarbeiter eines EP befähigt werden, eigene Entscheidungen hinsichtlich eines Entwicklungsprojekts im Umgang mit dem Kunden zu treffen und die entsprechenden Kompetenzen technischer Art, in Projektmanagement, Teamfähigkeit, Kommunikation und Verhandlung etc. besitzen. Positive Erfahrungen aus erfolgreichen Prozessentwicklungsprojekten führen zu verstärktem Vertrauen in die technischen und kommunikativen Kompetenzen des EP und zu erhöhter Kundenbindung. Dieses Vertrauen führt nicht zuletzt zu höherer Kundenbindung auch im Produktgeschäft eines EP. Ein erfolgreiches Dienstleistungsgeschäft fördert somit ebenfalls den Vertrieb der Produkte eines EP.

OP gründen häufig selbständige Unternehmen, die zuständig sind für Entwicklung, Vermarktung und Erbringung von Outsourcingdienstleistungen. Ein typisches Geschäftsmodell eines solchen OP besteht darin, Maschinen und Anlagen eines Kunden zu kaufen, diese für den Kunden zu betreiben und eine bestimmte Leistung aus dem Betrieb der Anlagen an den Kun-

\section{Tab. 3 Fähigkeiten zur Anpassung der Organisationsstruktur}

\section{Entwicklungspartner}

\begin{tabular}{|c|c|}
\hline Elemente & Ausprägungen \\
\hline $\begin{array}{l}\text { Organisatorische } \\
\text { Trennung des } \\
\text { Produkt- und Dienst- } \\
\text { leistungsgeschäfts }\end{array}$ & $\begin{array}{l}\text { - Spezielles F\&E-Team für Ent- } \\
\text { wicklungsdienstleistungen } \\
\text { - Regelmäßiger Wissens- } \\
\text { austausch zwischen Team } \\
\text { und zentraler F\&E } \\
\text { - Klar geregelte Verantwort- } \\
\text { lichkeiten für Kunden im } \\
\text { Team }\end{array}$ \\
\hline $\begin{array}{l}\text { Sichtbarkeit der } \\
\text { Dienstleistungs- } \\
\text { aktivitäten für den } \\
\text { Kunden }\end{array}$ & $\begin{array}{l}\text { - Sichtbare Kontaktpunkte für } \\
\text { externe Kunden in Bezug } \\
\text { auf Dienstleistungsangebot } \\
\text { - Klare Verantwortlichkeiten } \\
\text { für das Dienstleistungs- } \\
\text { geschäft } \\
\text { - Feste Meetingstrukturen } \\
\text { und gemischte Teams mit } \\
\text { Kunden während Entwick- } \\
\text { lungsprojekten }\end{array}$ \\
\hline
\end{tabular}

\section{Outsourcingpartner}

\begin{tabular}{|c|c|c|}
\hline Fähigkeiten & Ausprägungen & Fähigkeiten \\
\hline $\begin{array}{l}\text { - F\&E-Kompetenzen } \\
\text { - Systematisches Wissensma- } \\
\text { nagement (z. B. IT-Systeme) } \\
\text { - Wissensaufbau aus } \\
\text { Kundenprojekten } \\
\text { - Wissensaufbau aus eigenen } \\
\text { technischen Entwicklungen } \\
\text { im Produktgeschäft }\end{array}$ & $\begin{array}{l}\text { - Selbständiges Unternehmen } \\
\text { für Outsourcingdienstleis- } \\
\text { tungen } \\
\text { - Bildung eines neuen } \\
\text { Geschäftsmodells } \\
\text { - Zusammenarbeit mit } \\
\text { externen Parteien }\end{array}$ & $\begin{array}{l}\text { - „Architektonische“ } \\
\text { Kompetenzen zur } \\
\text { Geschäftsmodellbildung } \\
\text { - Risikoabschätzung und } \\
\text {-management } \\
\text { - Organisationales Lernens } \\
\text { - Unternehmerqualitäten } \\
\text { - Netzwerkkompetenzen }\end{array}$ \\
\hline $\begin{array}{l}\text { - Vermarkten und Erbringen } \\
\text { von Entwicklungsdienstleis- } \\
\text { tungen durch F\&E-Team } \\
\text { - Befähigung der Mitarbeiter, } \\
\text { Entscheidungen im Umgang } \\
\text { mit Kunden zu treffen } \\
\text { - Entscheidungsrelevante } \\
\text { Kompetenzen } \\
\text { - Interne und externe } \\
\text { Teamfähigkeiten }\end{array}$ & $\begin{array}{l}\text { - Reiner selbständiger } \\
\text { Dienstleistungsanbieter }\end{array}$ & $\begin{array}{l}\text { - Vermarkten und Erbringen } \\
\text { von Outsourcingdienstleis- } \\
\text { tungen }\end{array}$ \\
\hline
\end{tabular}


den zu verkaufen. Die Bildung eines solchen radikal neuen Geschäftsmodells erfordert von OP „,architektonische“ Kompetenzen (Henderson/Clark 1990, Tushman/Smith 2004). Manager eines OP müssen in der Lage sein, die Architektur oder Geschäftslogik der gesamten Wertschöpfungskette zu überblicken und abzuschätzen, wie sich Änderungen auf die Integration der Wertschöpfungsaktivitäten und die Beziehungen zwischen den Beteiligten auswirken. Damit verbunden sind notwendige Kompetenzen zu Risikoabschätzung und -management sowie die Fähigkeit des organisationalen Lernens (Slater/Narver 1995, Senge 1990). Für den OP erleichtert die organisatorische Trennung vom Mutterunternehmen zudem das Betreiben von Fremdprodukten. Für den Kunden ergeben sich aus einer Geschäftsbeziehung mit einem OP durch die frei werdenden Mittel neue Möglichkeiten zur Investition. Aus Sicht des OP verlangen die am Anfang stehenden Investitionen eine mehrjährige Laufzeit der Geschäftsbeziehung, um profitabel zu sein.

Die organisatorische Selbständigkeit und das hohe finanzielle Risiko machen typische Unternehmerqualitäten für das Management eines OP unerlässlich. Der Wandel zur organisatorischen Selbständigkeit erfordert zudem umfangreiche Zusammenarbeit mit externen Parteien. Die mehrjährigen komplexen Outsourcingprojekte sorgen für eine lange Kundenbindung und unterstützen die Bildung guter persönlicher Beziehungen zwischen OP und Kunden. Diese sind wichtig, um die Kundenbedürfnisse hinsichtlich der übernommenen Prozesse ausreichend zu verstehen und erleichtern es, Mitarbeiter des Kunden mit den Prozessen zu übernehmen. Für die hohen Anfangsinvestitionen und den Umgang der mit der Prozessübernahme verbundenen Risiken benötigen OP eine enge Zusammenarbeit mit Finanzinstituten und Versicherungen. Nicht zuletzt bestehen enge Verbindungen zum Mutterunternehmen z. B. was die Ersatzteilversorgung der übernommenen Anlagen und Maschinen angeht oder einen möglichen Querverkauf von Verbrauchsmaterialien. Die vielfältigen Beziehungen zu Externen erfordern spezielle Netzwerkkompetenzen, wie etwa Fähigkeiten zur Bildung von Informationssystemen zwischen den Beteiligten, zur Gestaltung von Produktions- und Logistikprozessen und zur Koordination der unterschiedlichen Geschäftsbeziehungen (Möller/Svahn 2003).

\section{Personalmanagement}

Das Personalmanagement hat eine entscheidende Rolle bei der Entwicklung zum EP. Zu Beginn setzt ein EP interne Mitarbeiter aus der F\&E für Entwicklungsdienstleistungen ein, aber bei entsprechendem Erfolg der Entwicklungs- und Konstruktionsdienst-

\section{Tab. 4 Fähigkeiten zur Anpassung des Personalmanagements}

\section{Entwicklungspartner}

\begin{tabular}{l} 
Elemente \\
\hline Personalrekrutierung \\
\hline
\end{tabular}

Personalentwicklung Ausprägungen

- Zu Beginn interne Mitarbeiter aus F\&E für Entwicklungsdienstleistungen

- Absolventen von technischen Hochschulen

- Projektingenieure von professionellen Engineering-Beratungen oder anderen Herstellern

- Teilweise zeitlich befristete

Zusammenarbeit mit Freelancern oder Ingenieurbüros

- Trainee-Programme

- On-the-job-Training

- Mentoring-Programme

Personalentlohnung

\begin{tabular}{l} 
Fähigkeiten \\
\hline - Technische Fähigkeiten \\
- Kommunikative Fähigkeiten \\
- Projektleitungs- \\
kompetenzen \\
- Kurzfristige Integration \\
Externer wenn erforderlich
\end{tabular}

- Technisches Anforderungsprofil erstellen

- Kundenorientierung

- Vertrauen und Commitment schaffen

- Fähigkeiten zur Interaktion mit Kunden

- Gegenseitiges Lernen mit und von Kunden

- Bilden von Kennzahlen für die Leistung des F\&EDienstleistungs-Teams

\section{Outsourcingpartner}

\begin{tabular}{l} 
Ausprägungen \\
\hline - Manager zu Beginn aus \\
internen Produkt- oder \\
Dienstleistungsbereichen \\
- Übernahme von Frontline- \\
Mitarbeitern vom Kunden \\
- Monetäre und nicht \\
monetäre Anreize
\end{tabular}

Fähigkeiten
- Manager:
Management- und
Unternehmerqualitäten
- Frontline-Mitarbeiter:
Anwendungs- und
Prozess-Know-how

- On-the-job-Training für kontinuierliche Entwicklung bezüglich der übernommenen Prozesse

\section{- Kommunikative Fähigkeiten im Umgang mit Kunden - Wissensaustausch zwischen übernommenen und bestehenden Mitarbeitern}

\footnotetext{
- System berücksichtigt Kennzahlen des Kunden

- Indikatoren wie Kundenzufriedenheit, Mitarbeiterzufriedenheit und finanzielle Kennzahlen
}

- Integrieren von Kennzahlen des Kunden 
leistungen kann der Bedarf nicht nur durch interne Mitarbeiter gedeckt werden. Der Zugang zu gut ausgebildeten externen Mitarbeitern ist relativ schwierig. Deshalb werden vielfach FreelanceIngenieure und Berater zeitlich befristet eingesetzt oder eine Zusammenarbeit mit Ingenieurbüros angestrebt. EP verfügen in der Regel über ein entsprechendes Portfolio externer Kontakte, auf die je nach Kundenauftrag zurückgegriffen werden kann. Bei der Personalrekrutierung und -entwicklung konzentrieren sich EP neben den technischen und kommunikativen Fähigkeiten ebenfalls auf Projektleitungskompetenzen, welche einen reibungsfreien Ablauf von Entwicklungsprojekten gewährleisten. Bei den kommunikativen Fähigkeiten ist es für technische Berater entscheidend, Kunden richtig zuhören bzw. verstehen zu können, so dass die Entwicklungsherausforderungen des Kunden relativ einfach in ein technisches Anforderungsprofil zu überführen sind. Dazu müssen technische Berater vorab erfassen können, wie die neuen Prozesse Kunden in deren Verhalten und Zielen einen Nutzen stiften. Die kundenorientierten kommunikativen Fähigkeiten eines technischen Beraters beinhalten zudem, Vertrauen und Commitment zwischen dem EP und seinem Kunden zu schaffen, sowie Fähigkeiten zur Interaktion mit dem Kunden, um in einer Beziehung gegenseitigen Lernens den gemeinsamen Entwicklungsprozess erfolgreich zu durchlaufen (Möller 2006). Hierfür werden meist Absolventen von technischen Hochschulen und Projektinge- nieure von professionellen Engineering-Beratungen oder anderen Herstellern eingestellt. Die Entwicklung der Fähigkeiten werden durch Trainee-Programme, On-the-job-Training und MentoringProgramme unterstützt, bei denen erfahrene Mitarbeiter ihre Kenntnisse sukzessive an jüngere Mitarbeiter weitergeben. Auch das Personalentlohnungssystem erfordert eine Änderung. Typischerweise wird es angepasst, indem die Leistung des neuen Teams für Entwicklungsdienstleistungen in die Kalkulation einbezogen wird. Die Kalkulation basiert in der Regel auf finanziellen Kennzahlen wie Umsatz und Gewinn durch die Projekte mit externen Kunden.

Beim Wandel zum OP finden weniger umfangreiche Anpassungen im Personalmanagement statt. Die Managementebene wird am Anfang meist mit Managern aus internen Produkt- oder Dienstleistungsbereichen besetzt. Auch die Personalentwicklung auf Mitarbeiterebene ist relativ unkritisch, da in der Regel die Mitarbeiter des Kunden übernommen werden. Intensive Schulungen zu Anwenderkenntnissen, Kommunikationsfähigkeiten und Kundenorientierung sind deshalb nur beschränkt notwendig. Zusätzlich kann das Streben nach Effizienz und Skaleneffekten beim OP zu einer hohen Spezialisierung von Mitarbeitern bei vergleichsweise geringen Qualifikationsanforderungen führen. Kritischer erscheint deshalb die Rekrutierung von Mitarbeitern des Kunden. OP nutzen vor allem monetäre und nicht monetäre Anreize, um

\section{Best- und Worst-Practices im Vertriebs- und Kundenmanagement. Mit Handlungsempfehlungen}

オ

WWW.GABLER.DE

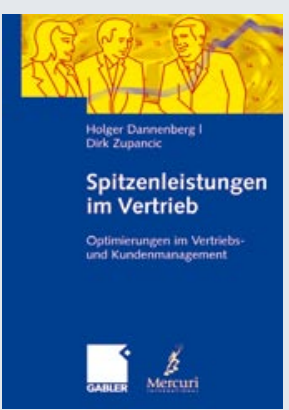

Holger Dannenberg / Dirk Zupancic

\section{Spitzenleistungen im Vertrieb}

Optimierungen im Vertriebs- und Kundenmanagement.

Mit Handlungsempfehlungen

2008. XXVIII, 234 S. Geb. EUR 52,00 ISBN 978-3-8349-0472-0

„Excellence in Sales“ ist ein integrierter Managementansatz für professionelle Vertriebsorganisationen. Das Konzept basiert auf einer weltweit einmaligen Befragung von 747 Unternehmen in 16 Ländern. Die Autoren analysieren Best- und Worst-Practices im Vertriebs- und Kundenmanagement. Echte Bestleistungen im Vertrieb werden anhand einer Reihe von Stellhebeln systematisch erläutert und durch Fallbeispiele ergänzt.

www.wirtschaftslexikon.gabler.de Jetzt online, frei verfügbar! フ

Einfach bestellen:

kerstin.kuchta@gwv-fachverlage.de Telefon+49(0)611.7878-626 
Mitarbeiter für einen Wechsel zu motivieren und langfristig an sich zu binden. Monetär sind dies z. B. höhere Gehälter oder ein jährlicher Bonus, nicht monetär z. B. zusätzliche Urlaubstage. Durch die Bindung der Mitarbeiter des Kunden ist es für OP relativ einfach, die bisherigen Leistungsindikatoren bei den übernommenen Prozessen zu erreichen. Ohne das entsprechende Anwendungs- und Prozess-Know-how dieser Mitarbeiter ist dies nur sehr schwierig möglich. Eine Erhöhung und Verbesserung der Leistungsindikatoren wird durch eine kontinuierliche Personalentwicklung bezüglich des übernommenen Prozesses gewährleistet. Diese Personalentwicklung basiert im Wesentlichen auf einem Wissensaustausch zwischen den übernommenen Mitarbeitern und den Wissensträgern innerhalb des OP. Die größten Anpassungen finden im Entlohnungssystem für einen OP statt. Beim OP verschwimmt das Entlohnungssystem mit den Kennzahlen des Kunden, so dass sich der variable Lohnanteil auf Grund der Zielerreichung der Kundenvorgaben errechnet. Ebenfalls berücksichtigt werden Indikatoren wie Kundenzufriedenheit, Mitarbeiterzufriedenheit und Erfolgskennzahlen wie die operative Marge (Heskett et al. 1997).

\section{Entwicklungs- und Outsourcingdienstleistungen bieten interessante strategische Stoßrichtungen für Investitionsgüterhersteller}

Kundenbedürfnisse nach individueller Prozessberatung und -entwicklung können für technologieorientierte Hersteller ein Anstoß sein, über das Anbieten von Entwicklungsdienstleistungen nachzudenken. Kundenbedürfnisse, sich stärker auf Kernaktivitäten zu fokussieren und Kapitalbindung zu reduzieren, lassen das Anbieten von Outsourcingdienstleistungen für Hersteller mit Anwendungskompetenzen attraktiv erscheinen. Beide Strategien erfordern hohes Vertrauen und enge strategische Partnerschaften mit Kunden. Dabei bestehen auch Gefahren, zu stark von einzelnen Kunden abhängig zu werden oder durch ausgewählte Partnerschaften andere Kunden zu verlieren. Die Entwicklung vom Hersteller zum Anbieter von Entwicklungs- oder Outsourcingdienstleistungen liefert interessante Erfolgsaussichten, erfordert jedoch weitreichende Konsequenzen für das Organisationsdesign und den Aufbau notwendiger organisationaler Fähigkeiten, die unbedingt berücksichtigt werden müssen.

\section{Literatur}

Backhaus, K./Kleikamp, C. (2001): Marketing von investiven Dienstleistungen, in: Bruhn, M./Meffert, H. (Hrsg.): Handbuch Dientleistungsmanagement: Von der strategischen Konzeption zur praktischen Umsetzung, Gabler, 2. Auflage, Wiesbaden.

Belz, C./Schuh, G./Groos, A./Reinecke, S. (1997): Industrie als Dienstleister, Thexis, St. Gallen.

Gebauer, H./Hildenbrand, K./Fleisch, E. (2006): Servicestrategien für die Industrie, Harvard Business Manager, Mai, S. 47-55.

Gummesson, E. (1994): Service Management: An Evaluation and the Future, International Journal of Service Industry Management, 5, 1, S. 77-96.
Henderson, R. M./Clark, K. B. (1990): Architectural innovation: the reconfiguration of existing product technology and the failure of established firms, Administrative Science Quarterly, 35, S. 9-31.

Heskett, J. L./Sasser, W. E./Schlesinger, L. A. (1997): The Service Profit Chain: How Leading Companies Link Profit and Growth to Loyalty, Satisfaction, and Value, New York: Free Press.

Homburg, C./Fassnacht, M./Guenther, C. (2003): The Role of Soft Factors in Implementing a Service-Oriented Strategy in Industrial Marketing Companies, Journal of Business-to-Business Marketing, 10, 2, S. 23-51.

Kleikamp, C. (2002): Performance Contracting auf Industriegütermärkten, Münster.

Kleinaltenkamp, M./Plötner, O./Zedler, C. (2004): Industrielles Servicemanagement, in: Backhaus, K./Voeth, M. (Hrsg.): Handbuch Industriegütermarketing, Gabler, Wiesbaden.

Lay, G./Schneider, R. (2001): Wenn Hersteller zu Serviceleistern werden, Harvard Business Manager, 23, 2, S. 16-24.

Matthyssens, P./Vandenbempt, K. (1998): Creating competition advantage in industrial services, Journal of Business \& Industrial Marketing, 13, 4/5, S. 339-355.

Möller, K. (2006): Role of competences in creating customer value: A valuecreation logic approach, Industrial Marketing Management, 35, S. 913-924.

Möller, K./Svahn, S. (2003): Managing strategic nets: A capability perspective, Marketing Theory Articles, 3, 2, S. 201-226.

Neu, W./Brown, S. (2005): Forming Successful Business-to-Business Services in Goods-Dominant Firms, Journal of Service Research, 8, 1, S. 3-17.

Noch, R. (1995): Dienstleistungen im Investitionsgüter-Marketing - Strategien und Umsetzung, FGM, München.

Pine II, B. J./Peppers, D./Rogers, M. (1995): Do You Want to Keep Your Customers Forever?, Harvard Business Review, 73, 2, S. 103-114.

Senge, P. (1990): The fifth discipline: The art and practice of the learning organization, New York: Doubleday Currency.

Slater, S. F./Narver, J. C. (1995): Market Orientation and the Learning Organization, Journal of Marketing, 59, 3, S. 63-74.

Tushman, M./Smith, W. (2004): Innovation streams, organization designs, and organizational evolution, in: Tushman, M./Anderson, P. (Hrsg.): Managing strategic innovation and change, New York: Oxford University Press.

\section{Die Autoren}

\section{Thomas Fischer}

Forschungsassistent und Doktorand am Institut für Technologiemanagement an der Universität St. Gallen. E-Mail:Thomas.Fischer@unisg.ch

\section{PD Dr. Heiko Gebauer}

Nachwuchsdozent für Technologiemanagement und Habilitand am Institut für Technologiemanagement an der Universität St. Gallen.

E-Mail: heiko.gebauer@unisg.ch

\section{Prof. Dr. Elgar Fleisch}

Professor für Informations- und Technologiemanagement an der Universität St. Gallen (HSG) und Direktor am dortigen Institut für Technologiemanagement sowie Professor für Informationsmanagement am Departement für Management, Technologie und Ökonomie der ETH Zürich.

E-Mail: Elgar.Fleisch@unisg.ch 


\section{Der Marketingberater für erfolgreiche Führungskräfte}

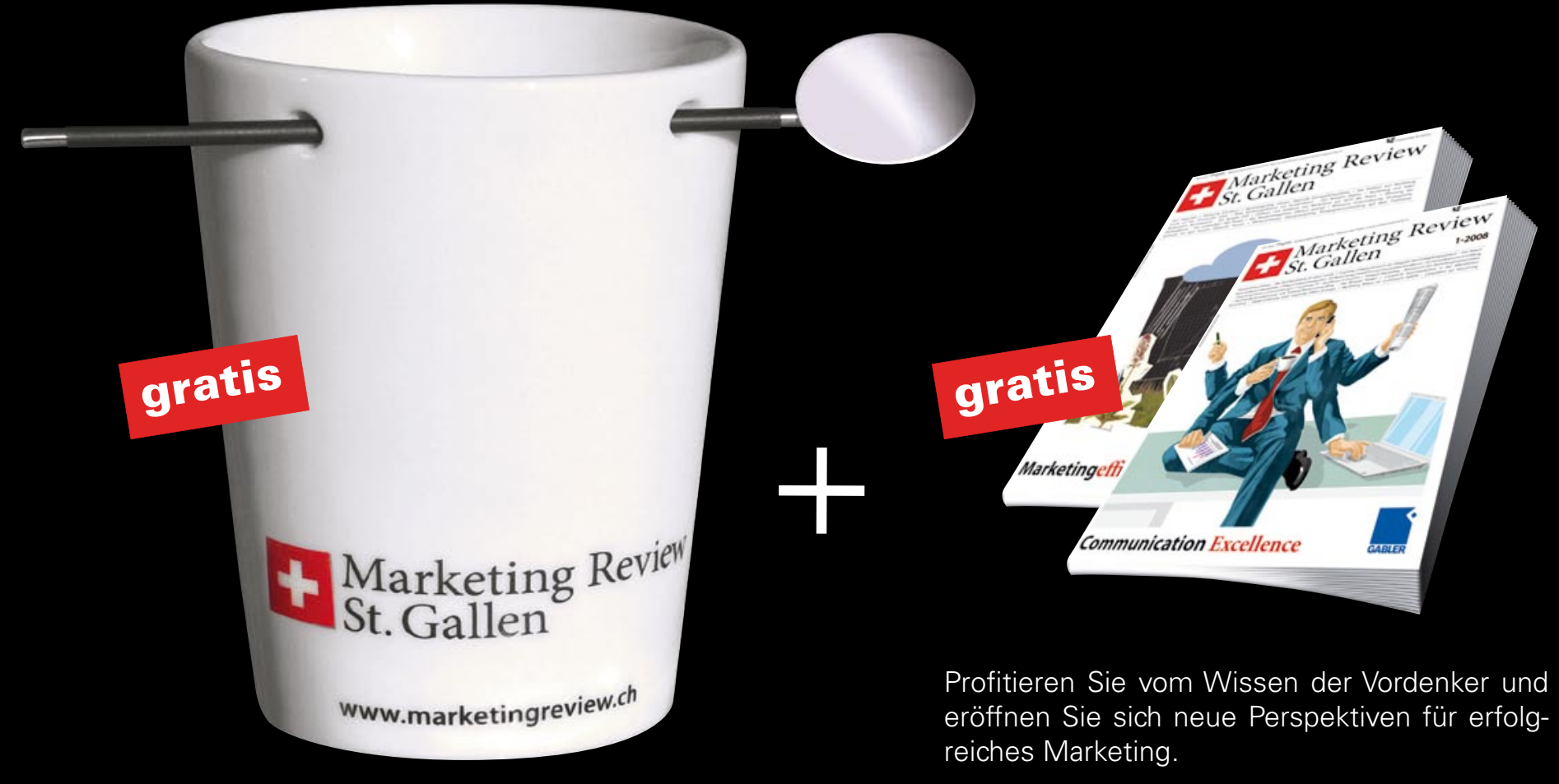

Einfach Fax-Gutschein ausftillen und faxen an: (0611) 7878407

JA, ich möchte 2 Ausgaben Marketing Review St. Gallen kennen lernen - plus die exklusive Tasse. Das Kennenlern-Paket erhalte ich gratis.

Name, Vorname

Firma, Abteilung

Straße, Nr.

PLZ, Ort
Oder bestellen Sie einfach

per Telefon:

+49 (0) 5241/801968

oder per E-Mail:

gabler@abo-service.info Wenn mir "Marketing Review St. Gallen" gefällt, brauche ben Marketing Review St. Gallen" zum Preis von € 155-inkl. Versand u. MwSt. (zahlbar gegen Rechnung). Den Bezug kann ich jederzeit zur nächsten erreichbaren Ausgabe beenden. Möchte ich Marketing Review St. Gallen" nicht weiter lesen, melde ich mich innerhalb von 10 Tagen nach Erhalt des 2. Probeheftes schriftlich. (Ein Angebot der GWV Fachverlage GmbH, Geschäftsführer Dr. Ralf Birkelbach, A.F. Schirmacher, AG Wiesbaden HRB 9754.)

Die Tasse und die Probehefte darf ich auf jeden Fall behalten. 